\title{
Analytical calculation of superconducting transition temperatures including a complete consideration of many-body interactions and non- equilibrium states
}

\author{
Shinichi Ishiguri, Ph.D \\ Nihon University \\ 1-2-1 Izumi-Cho, Narashinoshi, Chiba 275-8575 JAPAN \\ Email: shinichi.ishiguri@gmail.com
}

\begin{abstract}
In this work, we analytically describe a superconducting transition in a non-equilibrium state taking into account many-body interactions; the obtained transition temperatures indicate the presence of superconductivity at non-refrigeration temperatures.

First, we consider many-body interactions and discuss the case of locally thermal equilibrium with many-body interactions; in this section, we derive statistical equations that describe many-body interactions at locally thermal equilibrium state. Then, the same theory is used to derive a many-body statistical equation that is expanded to include the case of non-equilibrium states; in this case a transition temperature is derived. Moreover, a wave function of an EPR pair (Einstein-Podolsky-Rosen pair) is calculated according to the Lorentz conservation, and a specific condensation is observed and the Meissner effect is found to be present.

Furthermore, considering the Lorentz conservations, relativistic energy, and Boltzmann statistics, algorithms are presented to calculate charge density, current density, and internal local energy. We note that these calculations do not require a specific code but instead utilize the software Microsoft Excel.

We present plots showing the charge density and current density vs. the applied electric potential, which demonstrate the practical applicability of the theory. Moreover, internal local energy was found to be close to zero for sufficiently large electric potentials at non-refrigeration temperatures.

This paper describes non-equilibrium and EPR-pair type superconductivity, with the complete consideration of many-body interactions.
\end{abstract}

\section{Keywords}

non-equilibrium superconductivity, EPR-pair superconductivity, Many-body interaction, transition temperature, Lorentz conservations 


\section{Introduction}

Superconductivity was discovered approximately a century ago. The first discovery of superconductivity was in $\mathrm{Hg}$ refrigerated in liquid $\mathrm{He}(4.2 \mathrm{~K})$. This finding inspired significant research effort in the search for metal-based superconductors. Consequently, superconductors with higher critical temperature have been found, and superconductors with critical temperatures around $20 \mathrm{~K}$ have been discovered. The Bardeen-Cooper-Schrieffer theory [1], which was confirmed by much research work, limited the critical temperature of a superconductor to $30 \mathrm{~K}$. However, ceramic cuprates [2] that have critical temperatures higher than the boiling point of $\mathrm{N}_{2}(77 \mathrm{~K})$ have been discovered, which contravened the above theory that limited the presence of superconductivity to temperatures below $30 \mathrm{~K}$.

Since the discovery of ceramic cuprates, new compounds that have higher critical temperatures have been sought; this search has yielded Fe-based superconductors (with a critical temperature of $\sim 50 \mathrm{~K})[3], \mathrm{MgB}_{2}$ metal superconductors (39 K) [4], and H-based high-pressure superconductors $(>250 \mathrm{~K})$ [5]. We note, however, that $\mathrm{H}$-based superconductors have high critical temperatures but require extremely high pressures (above $250 \mathrm{GPa}$ ).

To date, compounds that have a critical temperature near room temperature $(300 \mathrm{~K})$ at ambient pressures have not been found. The discovery of these room-temperature superconductors would revolutionize the electrical and energy industries.

This study proposes a novel form superconductivity that does not require refrigeration. This superconductivity is generated in non-equilibrium states.

As another study about non-equilibrium states, Kubo theory [6] explains phenomena including electrical conductivity tensors in AC (Alternating current) states well. This theory can still be used to analyze a constant force at a constant temperature, but if the first perturbation approximation does not converge, Kubo theory cannot be applied. Furthermore, in addition the fact that this theory is relatively hard to use, it is known that this theory does not contain many-body interaction force positively.

To date very few previous studies generally have been able to solve many-body interactions analytically, and a limited number of non-equilibrium problems have been solved.

There are not many articles in the literature describing superconductivity in non-equilibrium states, although there are however special statistical theories and transitions [7-13]. We note that the method proposed in this work is applicable to many-body interactions. Thus far, many-body interactions have been resolved numerically, as in Ref. [14]. However, numerical calculations do not provide a physical insight into a problem, and thus it is preferable to solve many-body interaction problems analytically. The perturbation method [15] has been proposed, but it is merely calculated up to the finite term. That is, if the coverage in the perturbations is finite, the accurate calculations are difficult. In our previous 
work [16], we described many-body interactions and transition temperatures analytically.

The present study describes a further phenomenon in which there are both many-body interactions and non-equilibrium processes; in such situations the critical temperature of a superconductor can be higher than room temperature. We describe a novel form of superconductivity whose critical temperature is greater than room temperature, and we show the applicability of the proposed theory to both many-body interactions and non-equilibrium mechanics.

We note that the superconductivity considered in the present paper is similar to that presented in Ref. [17]. The Ref. [17] was described in terms of the divergent current density, but the present paper regards the applications of electrical potentials. However, EPR pair [18-19] superconductivity is common to both, which is novel with respect to existing superconductors.

\section{Methodology}

In this paper, we first present the setup used to generate non-equilibrium superconductivity. As shown in Fig. 1, two n-type semiconductor-samples, A and B, are prepared; each sample is a part of two independently prepared electric loops. A DC (direct current)-voltage source is connected between the systems A and B. This source is not a part of the two electric loops. This implies that the samples A and $B$ are subject to uniform electrostatic potentials. Given that each sample is subject to a uniform electrostatic potential, a current is generated in each system. The Lorentz conservation relation can thus be applied; from this relation, we find that an electron gains a momentum as follows,

$(\hbar k)^{2}-\left(\frac{e \varphi}{c}\right)^{2} \equiv 0$,

or,

$k= \pm \frac{e \varphi}{\hbar c}$

where $k, e, c$, and $\varphi$ denote the wavenumber, the charge of an electron, the speed of light, and the electrostatic potential, respectively. As can be seen from the above expressions, the current in the systems A and B will be opposite in direction to each other. Applying Kirchhoff's voltage law to each loop, we can then predict that each loop has no net voltage and that superconductivity will occur.

Generally, superconductivity is described in terms of the formation of Cooper pairs. In a later section of this work, the mechanism that generates this superconductivity will be seen to be novel; we note here that the together the systems A and B make up a so-called EPR pair [18-19]. That is, as described later, this type of superconductivity is explained via EPR pairs rather than Cooper pairs. 


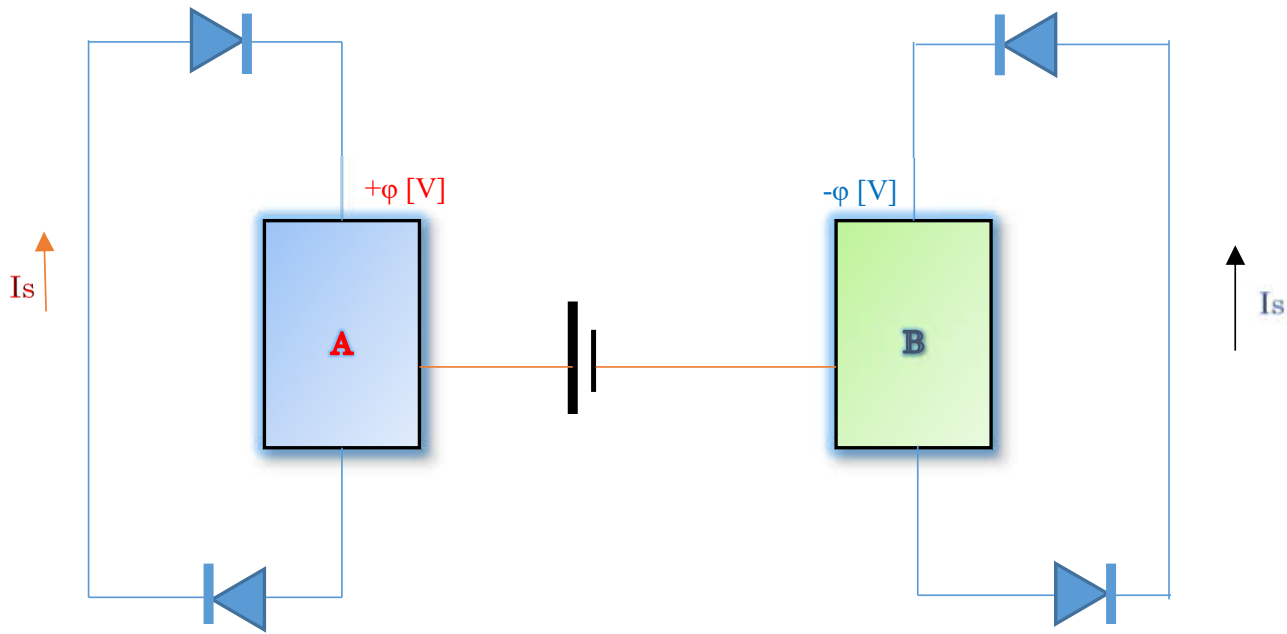

Fig. 1.

Schematic of the proposed system. The two electric loops are prepared and then a voltage source is used to connect these two electric loops. Due to this connection, each loop shows only electric potential (not a voltage). According to the Lorentz conservation, spontaneous currents $\pm I_{s}$ whose directions are opposite to each other must be generated. Thus, we can expect that each electric loop containing samples A and B have no electric resistance. Therefore, a condition of superconductivity is created. We note that the diodes are used to break the symmetry of the spontaneous superconducting current directions.

\section{Theory}

As discussed in Ref. [16], we review herein our established model to handle many-body interaction. Note that we refer many-body interaction without an asymmetry force as local thermal equilibrium and that many-body interaction with an asymmetry force as non-equilibrium. This reason will be clear later.

\subsection{The case of local thermal equilibrium without an asymmetric force}

The presence of a many-body interaction implies that there are many local temperatures, $T_{\mathrm{i}}$, at each point of the system (we note that the index $i$ denotes the location). As shown in Fig. 2, first we consider a spherical shell; at the center point of this spherical shell, there is a charged particle, such as an electron. In this model, a differential number $\mathrm{d} N$, has a local thermal equilibrium temperature, $T_{\mathrm{i}}$. The number of particles, $N$, is assumed to be sufficiently large to allow it to be treated as a continuous variable and thus $\mathrm{d} N$ can be defined. 


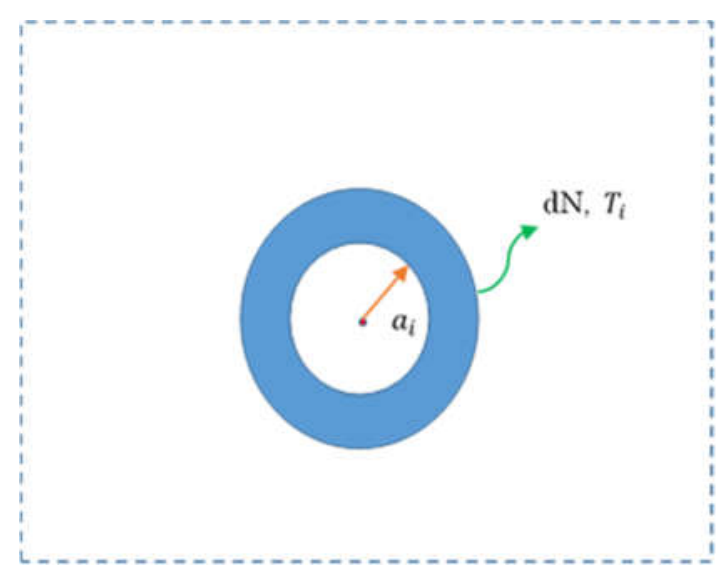

Fig. 2.

Schematic of the model used to analyze many-body interactions. When considering many-body interactions, the temperature has a spatial dependence. This model implies that a carrier exists at the center of the shell and $\mathrm{d} N$ takes a temperature $T_{i}$; thermal equilibrium can be assumed. Therefore, a force balance between force causing expansion due to the Coulomb interactions and force of compression due to the kinetic energies in $d N$ (i.e., the temperature $T_{i}$ ) is formed. By calculating this balance equation, one can obtain a novel statistical equation.

Here, we derive the statistical expression to handle many-body interactions.

We consider the forces that tend to cause an expansion in the spherical shell, as shown in Fig. 2, and the force that is compressive in nature; these forces must be balanced with each other at $\mathrm{d} N$.

The expansion force can take various forms depending on the specific case; the compressive force is assumed to be determined by the temperature, $T_{\mathrm{i}}$, and the value of $\mathrm{d} N$. We note that this case takes the Coulomb interaction as the expansion force. Thus, the force balance in this case can be stated as, (Coulomb interaction energy) $=\frac{3}{2} k_{B} T_{i} d N$, where $\mathrm{k}_{\mathrm{B}}$ denotes the Boltzmann constant.

As mentioned, the left-hand side is an expansion force, whereas the right-hand side is compressive in nature.

To determine the force balance equation, the Coulomb interaction energy and $\mathrm{d} N$ should be calculated. First, $\mathrm{d} N$ is represented as,

$\mathrm{dN}=\operatorname{gfd} \vec{k}=g f\left(\frac{1}{d v}\right)$,

where $\boldsymbol{k}, v, g$, and $f$ denote wave number vector, volume, state number, and partition function of particles, respectively.

This expression implies that within the spherical shell $\mathrm{d} N$ thermal equilibrium is reached. The electrostatic energy (i.e., the Coulomb interaction energy), $U_{\mathrm{E}}$, is calculated as, 
$U_{E}=\frac{1}{2} \varepsilon_{0}\left(\frac{e}{4 \pi \varepsilon_{0} a_{i}^{2}}\right)^{2} d v$

where $\varepsilon_{0}$ and $\mathrm{a}_{\mathrm{i}}$ denote the permittivity in the vacuum and the radius of a sphere shell $\mathrm{dN}$, respectively. The volume element of the integral can be expressed as, $\mathrm{d} v=\frac{1}{\mathrm{~d} \vec{k}}=\frac{4 \pi}{3} a_{i}^{3}$.

Substituting this volume element, the principle equation is calculated in terms of $\mathrm{d} N$ and $U_{\mathrm{E}}$. We find following equation is satisfied,

$a_{i}^{2}=\frac{9 \varepsilon_{0}}{e^{2}}\left(3 k_{B} T_{i}\right) f g$.

The particle that is subject to the many-body interactions considered here forms a stationary wave, and the wave function of the stationary wave and the eigenvalue (i.e., kinetic energy) are determined by the radius, $a_{i}$. As shown in Fig.3, the particle at the center is equivalent to a particle in an infinite well potential. Thus, the eigenvalue and wave function of this particle can be expressed as [20],

$$
\begin{aligned}
\psi_{i}(r) & =\sqrt{\frac{2}{2 a_{i}}} \sin \left(\frac{i \pi r}{2 a_{i}}\right), \\
E_{i} & =\frac{1}{2 m}\left(\frac{\hbar i \pi}{2 a_{i}}\right)^{2},
\end{aligned}
$$

where $m$ and $r$ denote the mass of a particle and the radial coordinate of the spherical coordinate system, respectively.

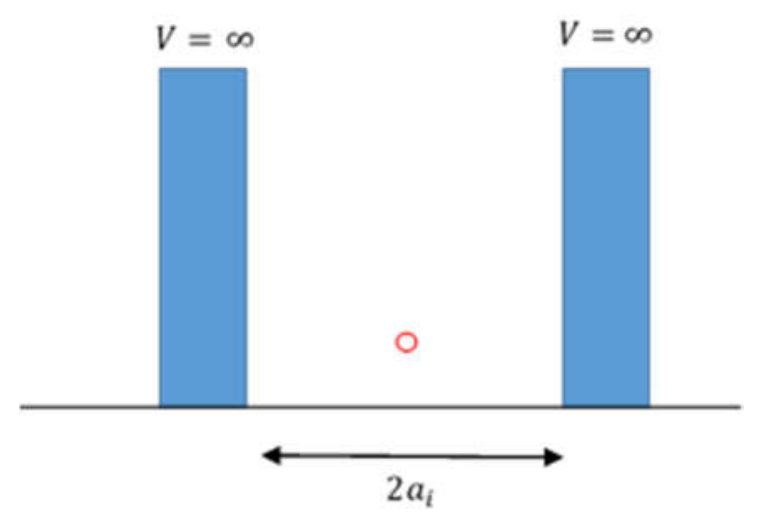

Fig. 3.

The model of the infinite well potential corresponding to the model shown in Fig. 2. The diameter $2 a_{i}$ varies, depending on the local temperature, $T_{i}$. A particle in this well potential takes the form of a stationary wave, and its wave function and eigenvalue are obtained from the literature. All of the parameters describing the well potential are dependent on the index $i$. 


\subsection{Applications to systems in local thermal equilibrium without an asymmetric force}

This section confirms the applicability of the model proposed in the previous subsection to problems of many-body interactions. Here, prior to the calculation of non-equilibrium statistics, let us investigate the case of the local thermal equilibrium without an asymmetric force. We first calculate the transitions temperatures of a ferromagnetic material, i.e., the Curie temperature.

We first discuss the background to emphasize the significance of this review and to confirm our new model. Many studies have been reported related to transition phenomena concerning ferromagnetic materials. Of these, the Ising model is the most widely used. Few studies exist, however, that accurately predicted accurately transition temperatures obtained in experimental work. Numerous statistical physics works describe that the two-dimensional (2D) Ising model predicts transition temperatures but the three-dimensional Ising model does not. Following the existing theory, a calculation of the transition temperature implies the evaluation of an exchange interaction. However, this interaction is too abstract to be evaluated for all ferromagnetic materials. A general formula determining transition temperatures has not been obtained; this is because the partition function for many-body interactions cannot be mathematically calculated.

Employing our model for many-body interactions, this section predicts the actual values of transition temperatures, which agrees with experimental values. We note that the following calculations do not utilize numerical calculation or fitting methods.

As shown in Fig 4, a classical magnetic moment, $\vec{\mu}$, is located at the center of a spherical shell, $\mathrm{d} N$, that has a local temperature $T_{\mathrm{i}}$. Based on above, the following relationship holds,

(magnetic field interaction due to magnetic moments) $=\frac{3}{2} k_{B} T_{i} d N$.

The left-hand side of the above equation can be expressed as,

$$
-\vec{\mu} \cdot \vec{B} .
$$

A magnetic field, $\boldsymbol{B}$, can be expressed as,

$\vec{B}=-\frac{\mu_{0}}{4 \pi}\left[\frac{\vec{\mu}}{r^{3}}-\frac{3(\vec{\mu} \cdot \vec{r}) \vec{r}}{r^{5}}\right]$

where $r$ is radius of the spherical shell, $\mathrm{d} N$. 


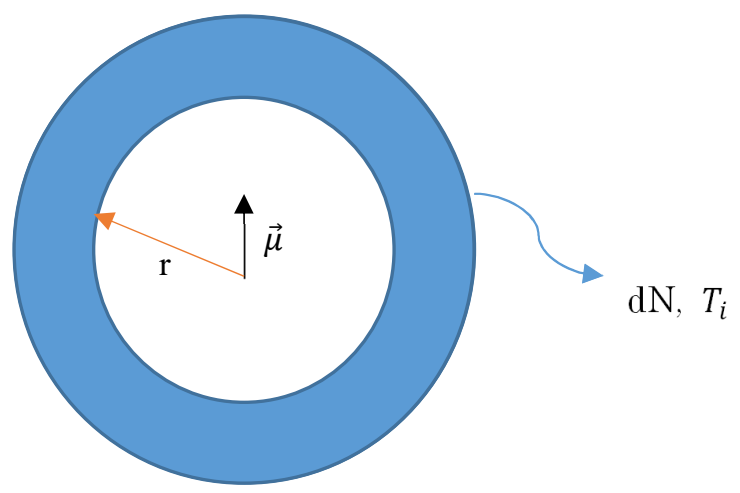

Fig. 4.

A schematic of the model applied to a ferromagnetic material. The force promoting expansion (from the central magnetic moment, $\vec{\mu}$,) is balanced by the compressive force from the region further from the center of the sphere, which is equal to net kinetic energies in the differential number $\mathrm{d} N$. Through the calculation of this force balance equation, we obtain a statistical equation that describes a many-body interaction.

The first and second terms of Eq. (10) describes ferromagnetism and antiferromagnetism, respectively. Because here we intend to calculate only ferromagnetic properties, this section considers only the first term from this equation. Furthermore, the orientations of the two magnetic moments $\vec{\mu}$ are assumed to be parallel i.e., the scalar product of the two $\vec{\mu}$ terms is positive. Thus, Eq. (10) becomes,

$-\vec{\mu} \cdot\left[-\frac{\mu_{0}}{4 \pi} \frac{\vec{\mu}}{r^{3}}\right]=\frac{3}{2} k_{B} T_{i} d N$.

By considering the volume element of the integral, the differential parameter $\mathrm{d} N$ can be represented by,

$$
\begin{aligned}
& \frac{\mu_{0}}{4 \pi}|\vec{\mu}|^{2} \frac{1}{r^{3}}=\frac{3}{2} k_{B} T_{i} d N=\frac{3}{2} k_{B} T_{i} \times g f d \vec{k}, \\
& \mathrm{~d} \vec{k}=\frac{1}{d v}=\frac{1}{\frac{4 \pi}{3} r^{3}}
\end{aligned}
$$

Thus, we obtain,

$$
\mu_{0}|\vec{\mu}|^{2}=\frac{9}{2} k_{B} T_{i} g f=\frac{9}{2} k_{B} T_{i} g \frac{1}{\exp \left(-\frac{E_{i}-E_{F}}{k_{B} T_{i}}\right)-1} .
$$

Note that the, in this equation, the Bose statistics was employed. In this Bose statistical equation, $E_{\mathrm{i}}$ denotes the zero-point energy of a phonon, i.e., the Debye temperature, and $E_{F}$ is a chemical potential that is equal to the Gibbs free energy. This energy describes only the internal energy. The chemical potential in the above equation is obtained from the electron specific heat coefficient, $\gamma$, as follows,

$$
\mu_{0}|\vec{\mu}|^{2}=\frac{9}{2} k_{B} g \frac{T}{\exp \left[-\frac{1}{k_{B} T}\left(\frac{3}{2} k_{B} \theta_{D}+\gamma T^{2}\right)\right]-1} .
$$

In the present case, the transition temperature, $T_{\mathfrak{c}}$, is assumed coincident with the extremum of Eq. 
(14). Therefore, when calculating the differentials, the local temperature, $T_{\mathrm{i}}$, should be considered to be a continuous variable representing the temperature, $T$, because there are now no parameters dependent on the index $i$, except $T_{i}$. Thus, the following equation can be derived:

$\frac{d}{d T} \mu_{0|\vec{\mu}|^{2}}=0$

Thus, we obtain,

$\frac{\gamma T^{2}-\frac{3}{2} k_{B} \theta_{D}}{k_{B} T}=-1$

$\mathrm{T} \equiv T_{C}=-\frac{k_{B}}{2 \gamma}+\sqrt{\frac{3 k_{B \theta_{D}}}{2 \gamma}} \approx \sqrt{\frac{3 k_{B \theta_{D}}}{2 \gamma}}$.

As an example, Table 1 lists the physical constants of the ferromagnetic metal, Fe.

Table 1. Physical constants of Fe.

Debye temperature, $\theta_{D} \quad 470 \mathrm{~K}$

Electron specific heat coefficient, $\gamma \quad 8.4 \times 10^{-27} \mathrm{~J} / \mathrm{K}^{2}$

Using these physical constants, the transition temperature, $T_{\mathrm{c}}$, of Fe can be calculated as,

$T_{c} \approx 1.08 \times 10^{3} \mathrm{~K}$.

Measurements of the transition temperature find it to be $1043 \mathrm{~K}$; this is in good agreement with the above result.

Next, the transition temperature of $\mathrm{Ni}$, which is ferromagnetic, is considered. $\mathrm{Ni}$ is much less thermally conductive than Fe. This suggests that a chemical energy (i.e., the internal thermal energy) may be neglected. Thus, from Eq. (16-1), the expression for $T_{\mathrm{c}}$ can be written,

$\frac{3}{2} k_{B} \theta_{D} \approx k_{B} T_{c}$.

Since the Debye temperature, $\theta_{\mathrm{D}}$, of Ni is reported as $450 \mathrm{~K}, T_{\mathrm{c}}$ can be found to be,

$T_{c} \approx 675 \mathrm{~K}$.

The measured transition value of $\mathrm{Ni}$ is $627 \mathrm{~K}$; thus, we find good agreement between the theory and experimental work.

\subsection{Application to non-thermal equilibrium in the presence of an asymmetric force}

Next, we consider the application of the present theory to non-thermal equilibriums subject to an asymmetric force, $F_{0}$. Our model is also applicable to many-body interactions and non-equilibrium situations. In this case, we consider that the spherical shell itself (not a particle) moves in the direction corresponding to the applied force $F_{0}$. Thus, the spherical shell radius, $a_{\mathrm{i}}$, must be converted to a 
wavelength (i.e., a wave number), which is written in terms of a center-of-mass motion; this motion is driven by the applied force $F_{0}$. Thus, in a non-equilibrium state, a spherical shell of radius $a_{i}$, is described by wavelength of center-of-mass motion induced by the applied force.

\subsection{Derivation of the superconducting transition temperature $T_{c}$ in non-equilibrium problems}

In this section, because we consider non-equilibrium states, the corresponding center-of-mass motion must be considered. This section employs the following motion equation,

$F_{0}=\hbar \frac{d k_{g}}{d t}$,

where $F_{0}$ is an external and asymmetric force, which is the origin of the non-equilibrium state of the system, and $k_{g}$ and $t$ represent the wavenumber along the center-of-mass motion and the time, respectively.

In this non-equilibrium system, the spherical shell moves due to the external force $F_{0}$. It is not considered that the carrier alone (for example, the electron) moves due to the force. This suggests that the radius of a spherical shell becomes a wavelength that is dependent on the center-of-mass motion,

$a_{i} \equiv 1 / k_{g}$,

where $k_{\mathrm{g}}$ denotes the wavenumber of the center-of-mass motion.

Thus, combining this equation of motion with Eq. (6), we obtain,

$\left(\frac{F_{0}}{\hbar} t\right)^{-2}=\frac{9 \varepsilon_{0}}{e^{2}} 3 k_{B} T f g$,

where,

$\mathrm{g}=\frac{1}{3 \pi^{2}}\left(\frac{2 m}{\hbar^{2}}\right)^{3 / 2} E^{3 / 2}$,

where $E$ denotes the kinetic energy, which is related to the force, $F_{0}$. Fermi statistics can be approximated by,

$f=f_{0} \exp \left(-\frac{E-E_{F}}{k_{B} T}\right)$

where $E_{\mathrm{F}}$ denotes the Fermi energy.

The above approximated partition function implies that n-type semiconductors are considered in this model. Substituting $f$ and $g$ into Eq. (21), the following expression can be calculated that implies the differential in terms of the temperatures. Thus, we can obtain,

$\frac{d t}{d T}=\frac{\beta \exp \left(-\frac{E-E_{F}}{k_{B} T}\right)\left[1+\frac{E-E_{F}}{k_{B} T}\right]}{2 t^{-3}\left(\frac{F_{0}}{\hbar}\right)^{-2}} \equiv 0$,

where,

$\beta=\frac{9 \varepsilon_{0}}{e^{2}} 3 k_{B} \frac{1}{3 \pi^{2}} f_{0}\left(\frac{2 m}{\hbar^{2}}\right)^{3 / 2} E^{3 / 2}$. 
From the above expression (24), the following equation is derived:

$1+\frac{E-E_{F}}{k_{B} T}=0$.

Noting that $T=T_{\mathrm{c}}$ at the transition point, the temperature will transition discontinuously to a temperature $\left|-\frac{|\Delta|}{k_{B}}\right|[\mathrm{K}]$ lower, as shown in Fig. 5. Note that $\Delta$ corresponds to an energy gap corresponding to the transition temperature $T_{\mathrm{c}}$.

When considering the transition temperature in Eq. (26) and because $E$ is the electrostatic potential energy, the fine structure constant $\alpha$ must be employed. The reason to utilize the fine structure constant $\alpha$ is as follows: According to Ref. [16], it is necessary to distinguish between a purely electric potential and an energy gap. The latter is converted to a photon, which has a momentum, whereas the former is not directly converted into a photon. That is, an energy gap can be expressed by two energy levels but a potential cannot. Thus, to convert an electric potential into an energy gap, the fine structure constant is required. Considering the above derived equation (26), we obtain the transition temperature $T_{\mathrm{c}}$ :

$T_{c}=\frac{E-E_{F}}{k_{B}} \alpha$,

where,

$\mathrm{E} \equiv|e \varphi|$.

The above equation (27) implies Eq. (24) indicates that the temperature changes discontinuously at time $t_{0}$, which is illustrated schematically in Fig. 5 .

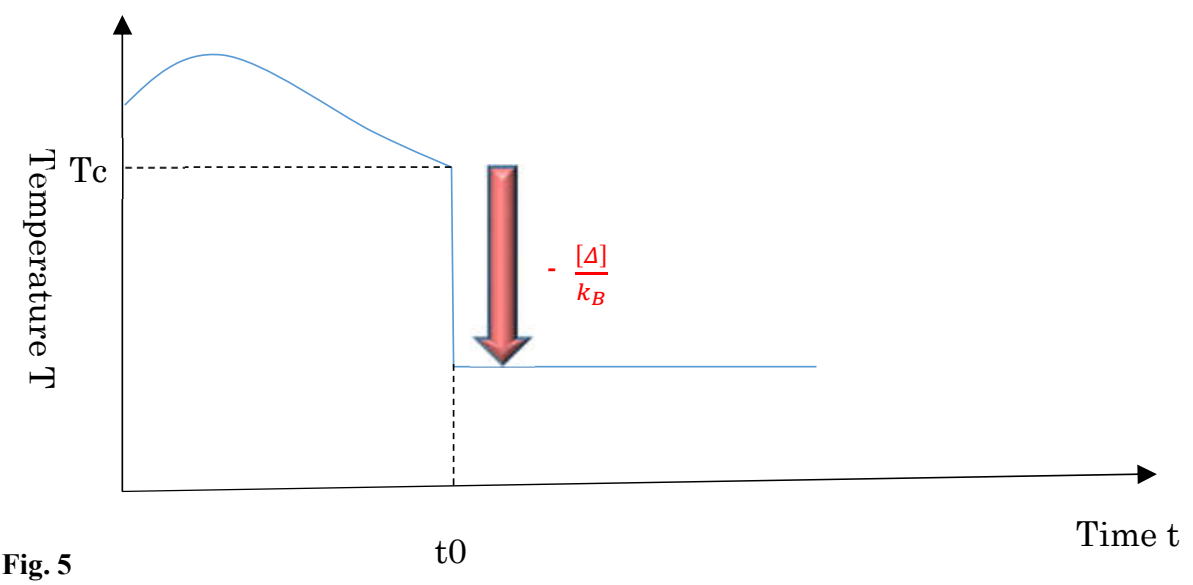

Schematic of a superconducting transition involving a non-equilibrium and many-body interaction. When the temperature is equal to $T_{\mathrm{c}}$, the energy discontinuously decreases in our proposed system. That is, our proposed 
system enables a material to undertake a superconducting transition. When interactions between particles are sufficiently strong, this work suggests that a phase transition under non-equilibrium conditions must occur.

\subsection{Mechanism of superconductivity}

Let us consider the mechanism of superconductivity. From the Lorentz conservation, the following equations hold,

$A^{2}-\left(\frac{\phi}{c}\right)^{2}=0$,

$(\hbar k)^{2}-\left[\frac{1}{c}(-e \phi)\right]^{2}=0$,

where $A$ denotes the vector potential and,

$\psi_{s}=\left|\psi_{s}\right| \exp (j k x)$,

$\int\left|\psi_{s}\right|^{2} d v=1$.

Note that these equations are independent of time (i.e., they are steady state expressions). Eq. (31-1) implies a plane-wave in which the wave number is derived from Eq. (30) and is also defined as a wave function by Eq. (31-2). We note that it is assumed that the normalization equation (31-2) is approximated as,

$\left|\psi_{s}\right|^{2}=k^{3}$.

In this equation (32), the inverse of volume element is assumed to be the wavenumber $k$.

Eq. (32) then yields,

$\left|\psi_{s}\right|^{2}=\left(\frac{e \varphi}{\hbar c}\right)^{3}$.

We now consider whether a macroscopic wave function is formed. As discussed, each electron has a wave function that can be expressed as,

$\psi_{s}=\left|\psi_{s}\right| \exp \left(-\frac{e x_{i}}{\hbar c} j \varphi\right)$.

We note that this wave function is valid only in one dimension. Considering the proposed system in this work, because electric potential $\varphi$ is arbitrary, the above derived equation (34) suggests that the wave number $k$ is also variable and constant along a given loop. Considering the uncertainty relation, this fact implies that the position vector $x$ must also be a constant. In the process of forming a condensation and as shown in Fig. 6, each position $x_{i}$ can be summed as scalars; each position (i.e., each state of an electron) is however independent and thus this does not contradict to the exclusion principle. These positions may only be added in one-dimension. It is possible to consider a total phase, $\Theta$, along the circuit A or B in Fig. 1. The total phase is given by Eq. (35) (without contradicting the exclusion principle):

$\Theta=\sum \frac{e \phi}{c \hbar} x_{i}=\frac{e \phi}{c \hbar} N x$ 
where $N$ is an integer.

$N x \equiv X$,

where $X$ implies the length along a loop A or B in Fig. 1 .
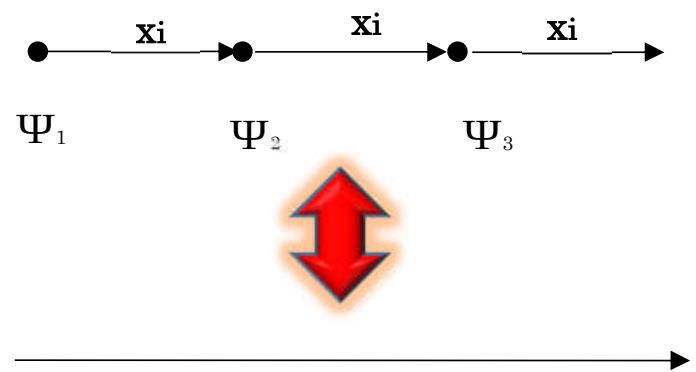

Fig. 6

$3 x i$

Schematic of the summation of each position vector in one-dimension. The quantum states of an electron must follow both Fermi statistics and the exclusion principle. In the present paper, however, the wave number is a function of the electrostatic potential $\phi$, which is arbitrary. i.e., the wave function is variable and constant along loops for which the electrostatic potential is constant. Although an electron (i.e., the wave function) takes each location that is indexed by $x_{i}$, the summation of all one-dimensional position vectors is possible. Consequently, it is suggested that position vector can be added without contradicting the exclusion principle (i.e., the upper panel is equivalent to the lower panel).

We can thus obtain the macroscopic wave function,

$\psi_{M}=\left|\psi_{M}\right| \exp \left(-\frac{e \phi}{c \hbar} X \mathrm{j}\right)$

$\int\left|\psi_{M}\right|^{2} d v=1$

We note that, because the shapes of the wave functions given in Eqs. (37-1) and (34-1) are same, $\left|\psi_{s}\right|^{2}=\left|\psi_{M}\right|^{2}$.

The macroscopic wave function suggests that condensation occurs with fermions along a loop.

Based on the previously mentioned Lorentz conservations of Eqs. (29) and (30), deleting the electric potential $\phi$ provides a relation between the vector potential $A$ and the wave number $k$,

$\mathrm{A}=-\mathrm{hk} \frac{c}{e}$.

From the probability flux equation given below in Eq. (40), a London equation (given in Eq. (41)) can be obtained. We note that, because we have obtained a macroscopic wave function, it is permitted to consider that the probability flux equation is formed.

$j_{q}=e\left|\psi_{M}\right|^{2} \frac{\hbar k}{m}$, 
$j_{q}=-\frac{e^{2}\left|\psi_{M}\right|^{2}}{m c} A$.

Thus, it can be concluded that the Meissner effect occurs in the proposed system.

\section{Method}

In this section, we consider simulations related to the above calculations. For the calculations in this work we use Microsoft Excel.

\subsection{Generated current and charge densities}

Here, we calculate the current and charge densities in the steady states.

From the Poisson equation, we have,

$\frac{d^{2} \Phi_{E}}{d r^{2}}=-\frac{\rho}{\varepsilon_{0}}$,

where $\Phi_{\mathrm{E}}, \varepsilon_{0}$, and $\rho$ denote a macroscopic electrostatic potential, the permittivity in the vacuum, and the charge density.

Under the condition that the charge density is constant, it can be found that,

$\rho=-2 \varepsilon_{0} \frac{\Phi_{E}}{r_{0}^{2}}$

where $r_{0}$ denotes an arbitrary length; in this work, this length is however assumed to be radius of the circuit loops A and B shown in Fig. 1.

Because this study presents the Lorentz conservations (Eqs. (29) and (30)), the following Lorentz conservation also holds,

$j^{2}-(c \rho)^{2}=0$,

where $j$ is the current density.

Thus, the current density is found to be,

$\mathrm{j}= \pm 2 \mathrm{c} \varepsilon_{0} \frac{\Phi_{E}}{r_{0}^{2}}$.

\subsection{Numerical calculations of many-body interactions}

First, we note that an electron with rest energy takes the Boltzmann statistic within a small region; 
$N^{\prime} \mathrm{m} c^{2} \approx N_{0} m c^{2} \exp \left(-\alpha \frac{e \Phi_{E}, i-E_{F}}{k_{B} T_{i}}\right)$,

where $N$ and $N^{\prime}$ denote the different particle numbers, respectively

Here, we make the following transformations; these transformations do not affect the generality of Eq. (46).

$N^{\prime} \rightarrow N_{0}$,

and,

$\mathrm{m} \rightarrow M_{1}$.

Thus, the particle $\mathrm{M}_{1}$ was created.

Furthermore, the particle $\mathrm{M}_{1}$ follows the Boltzmann statistics, thus

$M_{2} c^{2}=M_{1} c^{2} \exp \left(-\alpha \frac{e \Phi_{E}, i-E_{F}}{k_{B} T_{i}}\right)$.

In this equation, the particle $M_{2}$ is created. The above particles correspond to the creation of a spherical shell, as described in Sec. 3 .

The generalization of the above expression (47) yields,

$M_{i+1} c^{2}=M_{i} c^{2} \exp \left(-\alpha \frac{e \Phi_{E}, i-E_{F}}{k_{B} T_{i}}\right)$.

The above discussion valid for any variable index $i$ and thus we can compute results related to this section numerically.

In the following section, numerical results are provided.

\section{Result}

In this section, the Fermi energy is assumed to be $2.0 \mathrm{eV}$. In Fig. 7, the critical temperature, $T_{\mathrm{c}}$, is plotted against the applied electric potential $\varphi$. As shown, up to an electric potential of around $2 \mathrm{~V}$, thermal and equilibrium energy of the electrons inhibits the transition to a superconductive state. However, at temperatures that dominate over the electric potential of $2 \mathrm{~V}$, energy related to center-ofmass motion makes up a larger proportion of the total energy. Thus, a high critical temperatures are obtained in this situation. 


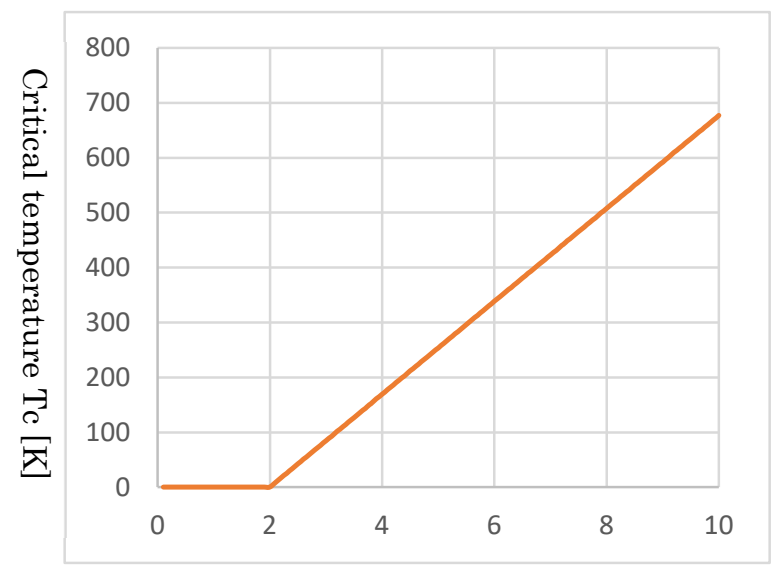

Fig. 7

Applied electric potential [V]

Critical temperature $T_{c}$ plotted against the applied electric potential. As shown, up to potential energies of around $2 \mathrm{~V}$, thermal and equilibrium energy of the electrons on the 2D surfaces is insufficient for the occurrence of superconductivity. However, for electric potentials larger than the electric potential of $2 \mathrm{~V}$, center-of-mass motion energy becomes larger than the energy of the thermal and equilibrium states. Thus, in this situation high critical temperatures are obtained.

In Fig. 8, the dependence of the current density on the electric potential is shown. As in the case of the critical temperature, for applied electric potential up to $2 \mathrm{~V}$, the current density is zero.

Afterward, however, it shows non-zero values. Because this change is non-continuous, it implies a superconducting transition.

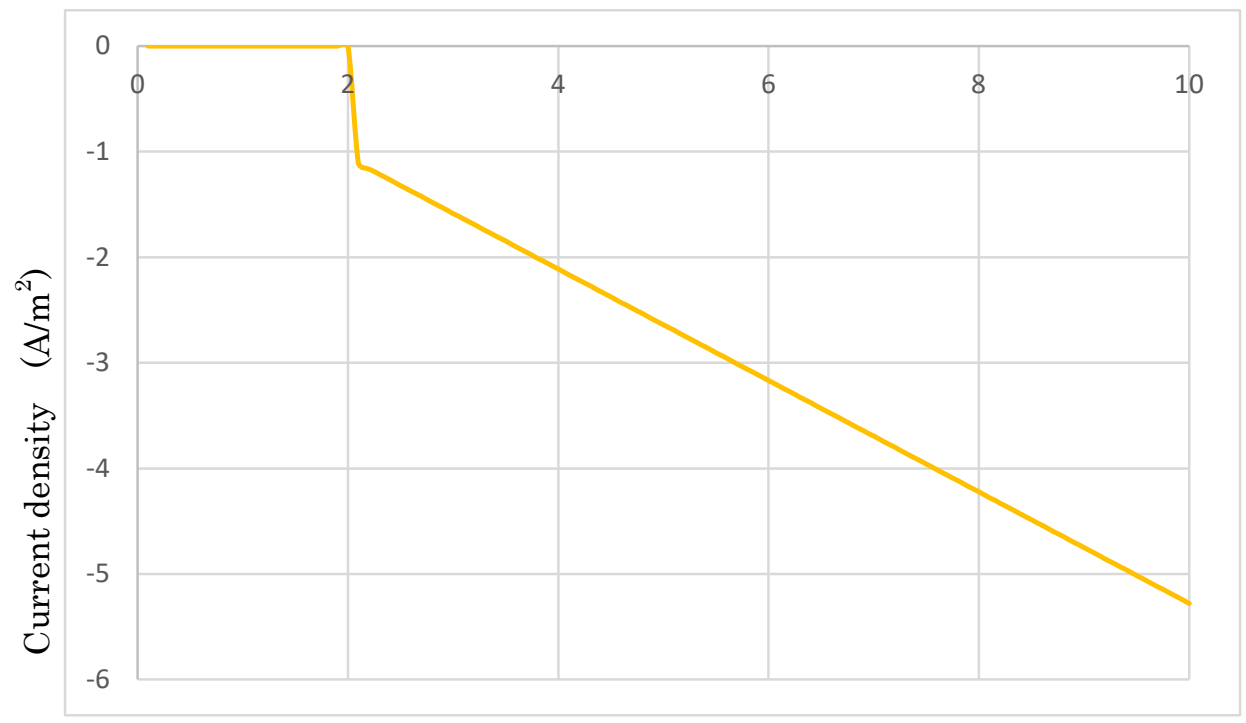

Applied electric potential [V] 
Fig.8

Current density vs. electric potential. Consistent with the obtained results for the critical temperatures, for applied electric potentials up to $2 \mathrm{~V}$, the current density is zero. However, for electric potentials greater than 2 $\mathrm{V}$, the current density is found to take non-zero values. This change is non-continuous and suggests a superconducting transition.

In Fig. 9, the charge density is plotted against the applied electric potential. The presented charge density is directly related to the current density obtained from the Lorentz conservation. Thus, the charge density curve is similar to the current density curve.

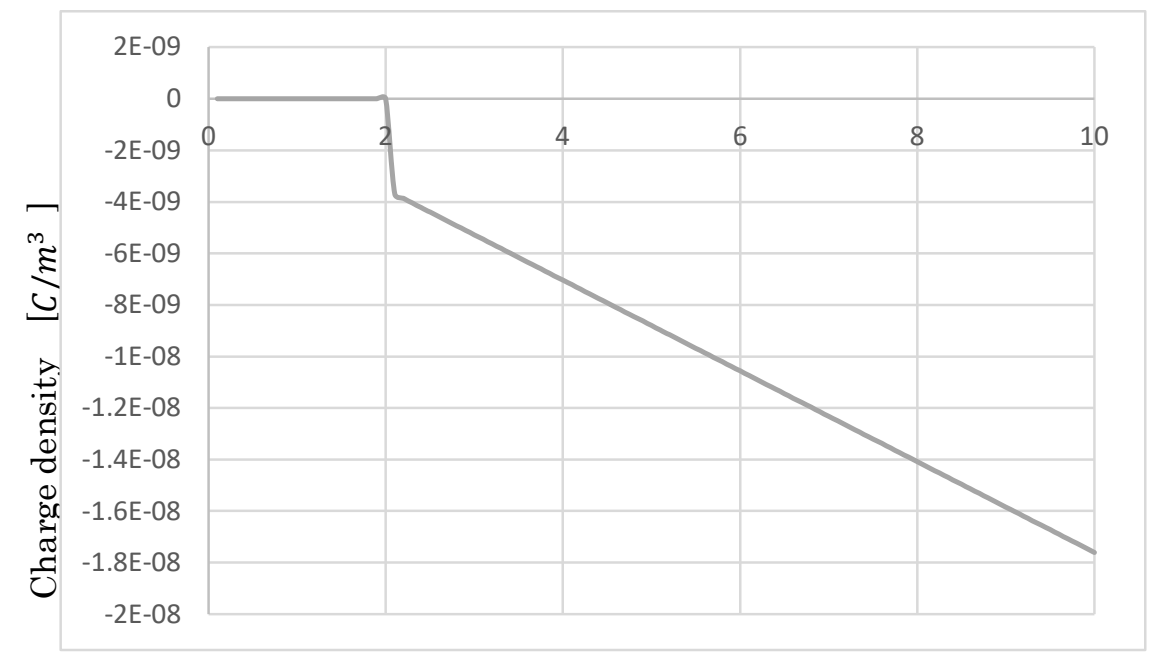

Fig. 9

Applied electric potential [V]

The charge density plotted against the applied electric potential. The charge density is directly related to the current density via the Lorentz conservation. Thus, this charge density behaves in a similar manner to the current density. Note that $r_{0}$ in Eq. (43) is assumed to be $10 \mathrm{~cm}$.

In Fig. 10, the current is plotted against the applied electric potential. The obtained curve is similar to that describing the current density. 


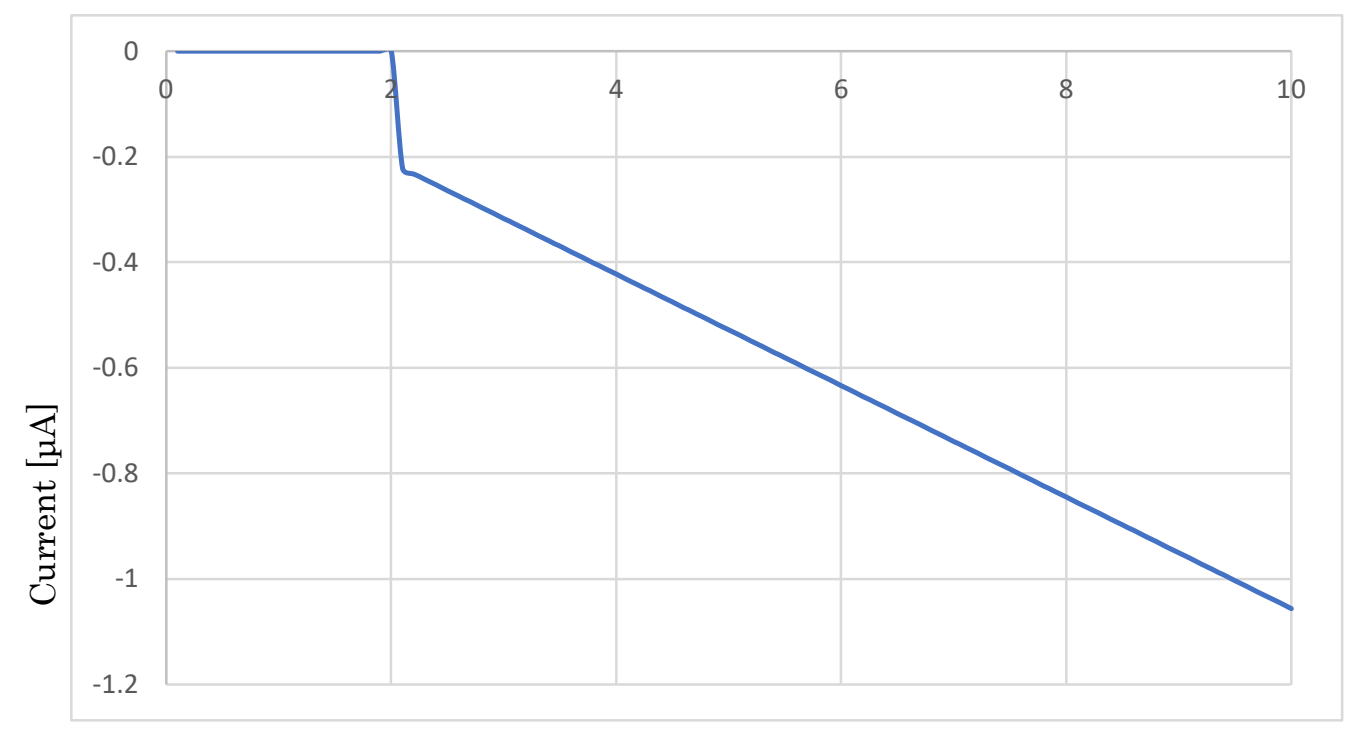

Applied electric potential [V]

Fig. 10

Current vs. applied electric potential. This curve is similar to that obtained for the current density. We note that the cross section of the leads in circuits $\mathrm{A}$ and $\mathrm{B}$ is assumed to be $0.2 \mathrm{~mm}^{2}$.

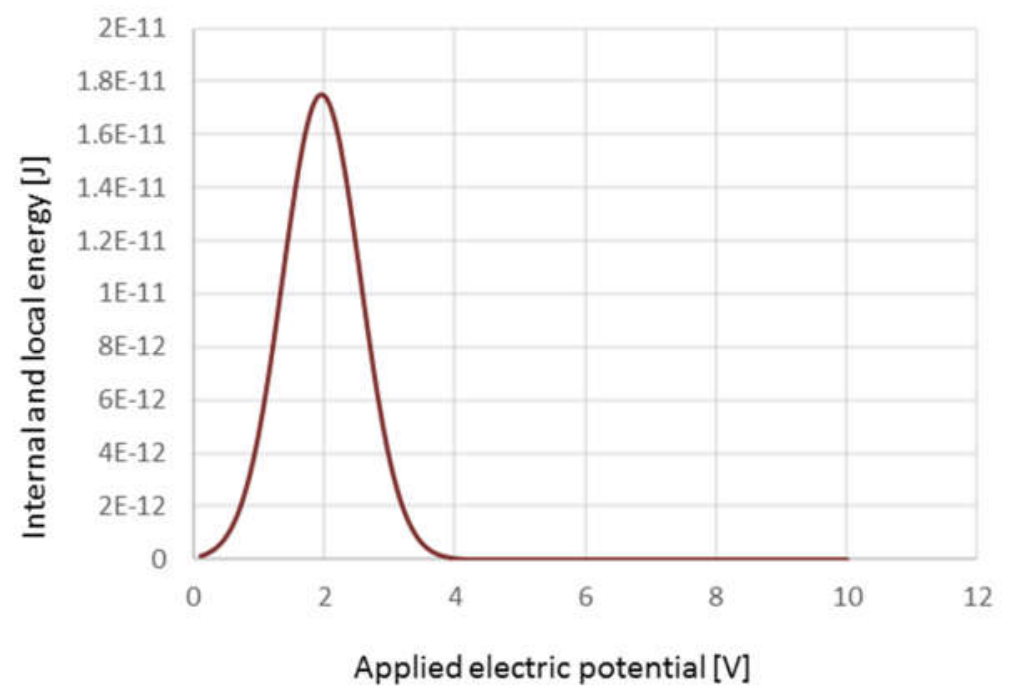

Fig. 11

Internal and local energy plotted against applied electric potential. As shown, at the Fermi energy the internal and local energy takes its maximum value. At $2.0 \mathrm{~V}$, the effect of interactions and many-body interactions are dominant; this implies that at this electric potential phase transitions are energetically favorable. 
In Fig. 11, the internal and local energies are plotted against the applied electric potential. In this figure, at the electric potential corresponding to the Fermi energy, the local and internal energies take their maximum values. At their maximum, very high local and internal energies are detected. This implies that, at that electric potential corresponding to the Fermi energy, the interaction energy and manybody effect take their maximum values. We thus infer that that the electric potential corresponding to the Fermi energy induces a phase transition.

In Fig. 12, the internal and local energies are plotted against temperature. The applied electric potential is kept constant at a value of $3 \mathrm{~V}$. Over around $50 \mathrm{~K}$, internal and local energies are close to zero. At around room temperatures, the internal and local energies remain zero; this implies that onedimensional condensation occurs. This indicates that a non-trivial phenomenon is occurring, which is a result of the many-body interactions.

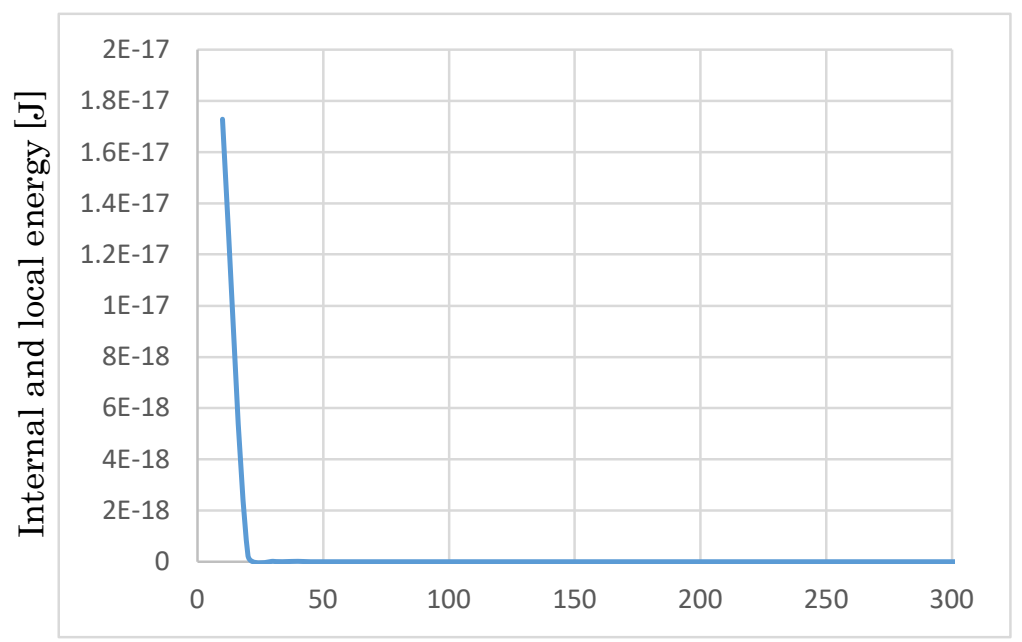

Temperature [K]

Fig. 12

Internal and local energies plotted against temperature. The applied electric potential is maintained constant at $3 \mathrm{~V}$. For temperatures greater than $50 \mathrm{~K}$, internal energy is close to zero. Thus, the internal energy is also zero close to room temperature; this implies that one dimensional condensation occurs. This finding indicates that a non-trivial phenomenon occurs. This phenomenon is a result from the many-body interactions. 


\section{Discussion}

\subsection{Why does the transition occur in the non-equilibrium states?}

It is necessary to distinguish the dimension in which the center-of-mass motion occurs and the other dimensions (i.e., 2-dimensional surface or area). In these other dimension (i.e., the surface), thermal equilibrium is still present. The total energy in all three directions must be conserved. Thus, when the momentum of center-of-mass motions increases, then the momentum in the remaining directions should decrease immediately after the maximum interactions effect. Thus, with the application of electric potentials a transition to a superconductive state can appear.

\subsection{What is mechanism of the superconductivity?}

Thus far it has been assumed superconductivity is generated by the formations of Cooper pairs. For example, the BCS theory claims that superconductivity requires the coupling of up- and down-spin electrons via phononic interactions. This Cooper pair is formed among the identical substances by a force, and then Bose-Einstein condensation occurs, which results in the Meissner effect. It is suggested, however, that there exists another source of superconductivity [17]; also in this case superconductivity results from one-dimensional fermion condensation. The mechanism of the superconductivity in this work originates from the condensation of one-dimensional fermions. Based on the Lorentz conservation, the phases of a wave function can be determined, which implies that the wavenumbers or position vectors are dependent on an electric potential. In the present paper, the position vectors along the electric circuit are summed into a single value and thus condensation occurs.

As discussed in the literature [17], this work indicates that the mechanism of superconductivity is also Fermi one-dimensional condensation below a critical temperature. We note that both wave functions in Ref. [17] and in the present paper are derived from the Lorentz conservation (i.e., from the theory of relativity) with the normalizations that define the wave functions as probabilities. However, as described in Section 3, in contrast with Ref. [17] where the wave numbers along an electric loop were summed to create single macroscopic wave number, in this work the positions along an electric loop are summed in order to provide single phase (i.e., the wave number is kept constant). We further note that in Ref. [17], the position vector was kept constant over the summed wave numbers.

An important point of this study is that this superconductivity is derived from electron pairs; these pairs do not form Cooper pairs but instead EPR pairs [18-19]. This situation is depicted in Fig. 1 in which a loop including sample A and another loop including sample B imply the existence of an EPR pair because interaction speed between them is of moment even though they are separated. 


\subsection{The significance of this paper}

There are several theories that describe quantum statistics. An important point is that, when considering fermions, only analytical approaches with a full treatment of many-body interactions can be used to obtain a phase transition temperature analytically.

Thermal equilibrium statistics for both fermions and bosons are widely studied in universities; these studies, however, do not consider the full nature of the interactions between particles. Thus, these conventional Fermi thermal equilibrium statistics fail to calculate a transition temperature analytically. To overcome this, several theories that handle many-body interactions have been presented, but they are numerical or fitting theories that also fail to calculate a transition temperature. In Ref. [16], the Curie temperatures of ferromagnetic materials were analytically calculated with a full consideration of the magnetic moments as many-body interactions. Moreover, in Ref. [16], using the principle of obtaining a full statistical expression with a complete consideration of macroscopic Bosons including many-body interactions, we succeeded in analytically calculating all the transition temperatures of high- $T_{c}$ cuprates.

Many researchers are investigating analytical formulations for statistical physics at thermal equilibriums with a full consideration of the many-body interactions. These theories are restricted to perturbations to Feynman diagrams or numerical calculations. However, in the cases where the perturbations do not converge, these methods do not work. Thus, very few reports provide analytical and non-equilibrium statistics with a full consideration of the many-body interactions present.

On the other hand, there is the Kubo theory of non-equilibrium statistics. This theory is widely used in the field of spintronics. Although this theory can still be used in the case of a constant force and constant temperature, in cases where the first perturbation approximation cannot be applied, this theory does not work correctly. Furthermore, this theory does not consider the actual interaction of the real particles and the many-body interactions.

In short, very few current theories are able to solve many-body interactions analytically, and very few non-equilibrium situations have been solved.

We believe that this paper is the first to calculate the superconducting transition temperature related to non-equilibrium states with a full consideration of many-body interactions. Furthermore, the calculated critical temperatures of superconductivity are such that the superconductivity is present at room temperatures. Thus, this work suggests that our proposed device results in a new phenomenon. 


\section{Conclusion}

Using the principle of the spherical shell model, this paper describes non-equilibrium and EPR-pairtype superconductivity. The mechanism behind the phenomenon is found to be a single dimensional condensation with critical temperature greater than room temperature. This work suggests the presence of various phenomena, in particular, the Meissner effect was demonstrated.

In this work, we have demonstrated:

1. A transition temperature including non-equilibrium states with full consideration of many-body interactions that can be calculated analytically.

2. The transition temperature describing the onset of superconductivity is greater than room temperature.

As a follow-up, the work presented here requires experimental confirmation.

\section{References}

[1] J. Bardeen, L.N. Cooper and J. R. Schrieffer, Phys. Rev. 108,(5), 1175-1204 (1957)

[2] J.G. Bednorz and K.A. Müller, Zeitschrift für Physik B. 64, 189-193 (1986)

[3] Kamihara, et al, J. Am. Chem. Soc, 128 (31), 10012-10013 (2006)

[4] J. Nagamatsu, et al, Nature, 410, 63 (2001)

[5] M. Somayazulu, et al, Phys. Rev. Lett. 122, 027001 (2019)

[6] R. Abe, "Statistic Mechanics", Publications of Tokyo University, pp.197-pp.203 (2017)

[7] S. Miyashita, et al "Transition and critical phenomenon", Science-sha, (2011)

[8] J.M. Kosterlitz, J. Phys. C, 7, 1046 (1974)

[9] S. Miyashita, Proc. Jpn. Acad,. Ser. B86, 643 (2010)

[10] M. Mekata, The Physical Society of Japan, 41,12, 968 (1986)

[11] A. Yoshimori, J. Phys.Soc.Jpn, 14, 807 (1959)

]12] O. Kahn, "Molecular Magnetism", (VCII, 1993)

[13] S. Sachdev, "Quantum Phase Transitions" Cambridge Univ. Press (2011)

[14] R.G. Par and W. Yang, "Density-Functional Theory of Atoms and Molecules", Oxford Univ. Press (1989)

[15] R. Abe, "Statistic Mechanics", Publications of Tokyo University, pp. 121-pp.133 (2017)

[16] S. Ishiguri, "Analytical Descriptions of High Tc Cuprates by Introducing Rotating Holes and a New Model to Handle Many-Body interactions," Preprints 2020, 2020050105 (doi: 10.20944/preprints202005.0105.v2)

[17] S. Ishiguri, "Generation system for renewable energy density substantially higher than that of power stations and presentations of novel superconductivity" Preprints, 2020, 2020030257 (doi: 10.20944/preprints202003.0257.v1) 
[18] A.Einstein, et al, Phys. Rev. 47, 777 (1935)

[19] S. Ishiguri, "New quantum diodes with superconducting properties at non-freezing temperatures, and their computing applications", Preprints, 2020, 2020080577 (doi: 10.20944/preprints202008.0577.v2)

[20] S. Nomura, "Introduction of quantum mechanics" pp.102-pp.108, Corona Publishing co., LTD, Tokyo, Japan (2002)

\section{Additional information}

This paper is not related to any competing interests such as funding, employment and personal financial interests. Moreover, this paper is not related to non-financial competing interesting.

\section{Acknowledgement}

We thank Enago (www.enago.jp) for the English language review. 